and plan are documented, and the GP receives a same day fax. We now report our results for the calendar year.

Results 293 patients with suspicious scans were contacted through our virtual clinic, and of these $241(82 \%)$ were triaged to the most appropriate next test as day cases (69 (29\%) bronchoscopy, 50 (21\%) EBUS, 69 (29\%) PET scan, 26 (11\%) CT biopsy, 4 (1\%) FNA, 17 (7\%) pleural aspiration, 6 (2\%) bronchoscopy and pleural aspiration).

At these attendances, patients were reviewed by medical staff and the CNS who undertook the telephone assessment, and any relevant other assessments performed. Of the remainder, 38 $(13 \%)$ were triaged to a clinic for assessment, 2 (6\%) were admitted, and 2 (6\%) referred back to their GP for best supportive care.

Using this service we have been successful in targeting cancer resources onto patients subsequently diagnosed with lung cancer (79\%), we have improved our mean time to diagnosis to 19 days, with a histological rate of $89 \%$. Our 62 day breach rate is $6 \%$. The new process also provides a $42 \%$ cost saving for primary care.

Conclusions We have shown that the use of a virtual clinic can target and speed up the diagnostic pathway for patients with lung cancer. It also makes more efficient use of scarce NHS resources, by ensuring that patients only attend the hospital for necessary investigations.

We recommend the use of this innovative service to other clinicians charged with managing this common and distressing disease.

\section{P176 EPIDERMAL GROWTH FACTOR RECEPTOR (EGFR) MUTATION TESTING AND TREATMENT CHOICE IN ADVANCED NON-SMALL CELL LUNG CANCER (NSCLC): UK FINDINGS FROM A GLOBAL SURVEY}

1) Spicer, ${ }^{2 B}$ Tischer, ${ }^{3} \mathrm{M}$ Peters. 'Guy's Hospital, London, UK; ${ }^{2}$ Kantar Health, Munich, Germany; ${ }^{3}$ Concord Hospital, Sydney, Australia

\subsection{6/thoraxjnl-2015-207770.313}

Introduction International and UK guidelines recommend EGFR mutation testing should be performed for advanced non-squamous NSCLC and results should guide treatment decisions. This testing is an important component of standard care because matching mutations to specific TKI treatment can extend survival and improve quality of life compared to first-line chemotherapy.

Objectives To assess the prevalence of EGFR mutation testing, attitudes and barriers to testing, and how results affect choice of therapy among prescribers in the UK and internationally.

Methods We conducted an online representative survey of 562 prescribers in 10 countries (Canada, France, Germany, Italy, Japan, South Korea, Spain, Taiwan, UK and US) between December 2014 and January 2015, including 51 oncologists in the UK (Annals of Oncology, Volume 26, Supplement 1, 2015).

Results In the UK, prescribers stated that $77 \%$ of newly diagnosed patients with advanced NSCLC were tested for EGFR mutations, the same proportion as in Europe (77\%) and North America (76\%), but less than in Asia (92\%). The stated reasons for not testing included: histology, insufficient tissue to perform the test, poor performance status, and long turnaround time for results. Despite the relatively high rate of EGFR testing claimed in the UK, respondents said that $21 \%$ of patients for whom a test was ordered were started on first-line treatment before results were available, nearly double the rate in Asia (12\%). Further, $67 \%$ of UK of oncologists, compared to $51 \%$ of all respondents, reported that treatment decisions were not affected by EGFR mutation subtype.

Conclusion Despite the high levels of EGFR mutation testing in the UK, the survey found that more than one in five patients with advanced NSCLC do not receive treatment personalised for cancer type and mutation subtype, even though evidence shows this improves survival and quality of life. These findings suggest there is incomplete implementation of UK guidelines. Further research is needed to discover what factors contribute to oncologists not following established guidelines in the UK. These data were originally presented at the European Lung Cancer Conference, 2015. Annals of Oncology, Volume 26, Supplement 1, 2015.

\section{REFERENCE}

1 Spicer J, Tischer B, Peters M. EGFR mutation testing and oncologist treatment choice in advanced NSCLC: global trends and differences. Annals of Oncology 2015;26(supplement 1):i57-i61

\section{Managing pleural disease}

\section{P177 COST EFFECTIVENESS OF AMBULATORY MANAGEMENT OF SPONTANEOUS PNEUMOTHORAX}

C Pillay, B Shah, M Naeem, R Reddy. Kettering General Hospital, Kettering, UK

10.1136/thoraxjnl-2015-207770.314

Introduction Hospital admissions due to a primary diagnosis of spontaneous pneumothorax (SP) have an annual incidence of 11.2 per $100000 .{ }^{1}$ It is estimated there are around 7200 emergency hospital admissions annually for pneumothorax in NHS. We have previously demonstrated pneumothorax patients requiring intercostal drains can be managed safely as outpatients with a Pneumostat device (similar to Heimlich valve). ${ }^{2}$

Methods All patients with primary spontaneous pneumothorax (PSP) and secondary spontaneous pneumothoraces (SSP) with a good performance status (WHO scale of $0-1$ ) requiring an intercostal drain were eligible for outpatient management. SP patients presenting to hospital between July'14 and June'15 were analysed to see what percentage could be managed on the ambulatory pathway. The number of bed days saved was calculated from the total number of days patients spent in the community with the chest drain. The savings were then extrapolated to whole of NHS.

Results 50 episodes (in 44 patients) of SP presented to hospital between July'14 and June'15. 36 episodes required a chest drain insertion. 20 of these 36 episodes (55\%) were managed on the ambulatory pathway. Based on this approximately 4000 (55\% of total NHS admissions) SP patients could be managed in an ambulatory setting annually across NHS.

The health care usage (number of attendances at ambulatory care, Chest X-rays and Pneumostat devices used) of patients managed on the ambulatory pathway is listed in table 1 . The unit cost of healthcare usage was obtained from the hospital information department and the overall cost of ambulatory care was calculated. The difference between this and the calculated bed day savings established the overall cost savings (Table 1). The potential savings to NHS equate to around $£ 2.8$ million based on the calculated average savings of $£ 703$ /patient.

Conclusion Ambulatory management of Spontaneous pneumothorax could realise significant savings to the NHS. 